\title{
Article \\ Clinically Relevant Escherichia coli Isolates from Process Waters and Wastewater of Poultry and Pig Slaughterhouses in Germany
}

\author{
Mykhailo Savin 1,2,*, Gabriele Bierbaum ${ }^{3}$, Judith Kreyenschmidt ${ }^{1,4}$, Ricarda Maria Schmithausen ${ }^{2}(\mathbb{D}$, \\ Esther Sib ${ }^{2}$, Silvia Schmoger ${ }^{5}$, Annemarie Käsbohrer ${ }^{5,6}$ (i) and Jens Andre Hammerl ${ }^{5, *}$ (i) \\ 1 Institute of Animal Sciences, University of Bonn, 53113 Bonn, Germany; j.kreyenschmidt@uni-bonn.de \\ 2 Institute for Hygiene and Public Health, Medical Faculty, University of Bonn, 53113 Bonn, Germany; \\ ricarda.schmithausen@ukbonn.de (R.M.S.); esther.sib@ukbonn.de (E.S.) \\ 3 Institute for Medical Microbiology, Immunology and Parasitology, Medical Faculty, University of Bonn, \\ 53113 Bonn, Germany; g.bierbaum@uni-bonn.de \\ 4 Department of Fresh Produce Logistics, Hochschule Geisenheim University, 65366 Geisenheim, Germany \\ 5 Department for Biological Safety, German Federal Institute for Risk Assessment, 10589 Berlin, Germany; \\ silvia.schmoger@bfr.bund.de (S.S.); annemarie.kaesbohrer@bfr.bund.de (A.K.) \\ 6 Department for Farm Animals and Veterinary Public Health and Epidemiology, Unit of Veterinary Public \\ Health and Epidemiology, University of Veterinary Medicine Vienna, 1210 Vienna, Austria \\ * Correspondence: s7mysavi@uni-bonn.de (M.S.); jens-andre.hammerl@bfr.bund.de (J.A.H.)
}

\section{check for}

updates

Citation: Savin, M.; Bierbaum, G.; Kreyenschmidt, J.; Schmithausen,

R.M.; Sib, E.; Schmoger, S.; Käsbohrer,

A.; Hammerl, J.A. Clinically Relevant

Escherichia coli Isolates from Process

Waters and Wastewater of Poultry and Pig Slaughterhouses in Germany. Microorganisms 2021, 9, 698 .

https://doi.org/10.3390/

microorganisms 9040698

Academic Editor: Kim Stanford

Received: 8 March 2021

Accepted: 25 March 2021

Published: 28 March 2021

Publisher's Note: MDPI stays neutral with regard to jurisdictional claims in published maps and institutional affiliations.

Copyright: (c) 2021 by the authors Licensee MDPI, Basel, Switzerland. This article is an open access article distributed under the terms and conditions of the Creative Commons Attribution (CC BY) license (https:/ / creativecommons.org/licenses/by/ $4.0 /$ )

\begin{abstract}
Escherichia coli is frequently associated with multiple antimicrobial resistances and a major cause of bacterial extraintestinal infections in livestock and humans. However, data on the epidemiology of (i) multidrug-resistant (MDR) and (ii) extraintestinal pathogenic E. coli (ExPEC) in poultry and pig slaughterhouses in Germany is currently lacking. Selected E. coli isolates $(n=71)$ with phenotypic resistance to cephalosporins from two poultry and two pig slaughterhouses expressing high MDR rates (combined resistance to piperacillin, cefotaxime and/or ceftazidime, and ciprofloxacin) of $51.4 \%$ and $58.3 \%$, respectively, were analyzed by whole-genome sequencing. They constituted a reservoir for 53 different antimicrobial resistance determinants and were assigned various sequence types, including high-risk clones involved in human infections worldwide. An ExPEC pathotype was detected in $17.1 \%$ and $5.6 \%$ of the isolates from poultry and pig slaughterhouses, respectively. Worryingly, they were recovered from scalding water and eviscerators, indicating an increased risk for cross-contaminations. Uropathogenic E. coli (UPEC) were detected in the effluent of an in-house wastewater treatment plant (WWTP) of a poultry slaughterhouse, facilitating their further dissemination into surface waters. Our study provides important information on the molecular characteristics of (i) MDR, as well as (ii) ExPEC and UPEC regarding their clonal structure, antimicrobial resistance and virulence factors. Based on their clinical importance and pathogenic potential, the risk of slaughterhouse employees' exposure cannot be ruled out. Through cross-contamination, these MDR E. coli pathotypes may be introduced into the food chain. Moreover, inadequate wastewater treatment may contribute to the dissemination of UPEC into surface waters, as shown for other WWTPs.
\end{abstract}

Keywords: E. coli; ExPEC; UPEC; virulence; MDR; resistance; slaughterhouse; wastewater

\section{Introduction}

The use of antimicrobials for veterinary purposes in Germany decreased over the years, encouraged by the nationwide antibiotics minimization concept in animal keeping. Between 2011 and 2017, a decrease of 57\% ( 973 tons) was observed in sales data. However, from 2015 onward, sold quantities of highest priority critically important antimicrobials (HPCIA, i.e., 3rd generation cephalosporins, fluoroquinolones, macrolides, polypeptides), which are particularly important for public health, stagnated or even slightly increased in the case of fluoroquinolones and polypeptides [1]. 
Because of the effects of cross-resistance and horizontal gene transfer, the consumption of antimicrobials belonging to HPCIA classes in veterinary medicine may contribute to the development, selection, and proliferation of resistance to clinically relevant antimicrobials [2,3]. Important examples are Enterobacteriaceae from the poultry and pig production chains that produce extended-spectrum $\beta$-lactamases (ESBLs). They are resistant to piperacillin and fluoroquinolones (3MDRO, multidrug-resistant Gram-negative organisms exhibiting resistance to three antibiotic groups ureidopenicillins, third/fourthgeneration cephalosporins and fluoroquinolones) [4-7]. Furthermore, Enterobacteriaceae carrying $\mathrm{pAmpC}$, bla $a_{\mathrm{OXA}-1}$ or with (hyper)production of inhibitor-resistant $b l a_{\mathrm{TEM}}$-variants, the occurrence of which is mostly reported for human clinical isolates, compromise the activity of $\beta$-lactam / $\beta$-lactamase inhibitor combinations (e.g., ceftolozane-tazobactam $(\mathrm{C} / \mathrm{T})$ and/or ceftazidime-avibactam (CZA)) [8,9].

Antimicrobial resistance has adverse consequences for human, animal, and environmental health ("One Health"). It was estimated that if no further action is taken, an annual death rate of 10 million humans may be reached by 2050 due to infections with multidrugresistant (MDR) bacteria [10]. Escherichia coli is often associated with multiple antimicrobial resistance and is one of the major causes of bacterial extraintestinal infections in humans, i.e., urinary tract infections (UTIs), infections of the skin and soft tissues, respiratory tract, meningitis as well as pneumonia [11]. In the poultry production system, pathogenic E. coli is also responsible for extraintestinal infections, collectively called colibacillosis, that are associated with the enormous economic loss [12]. Such strains belong to avian pathogenic E. coli (APEC). Escherichia coli strains responsible for extraintestinal diseases in humans are termed extraintestinal pathogenic E. coli (ExPEC). APEC and ExPEC can harbor diverse virulence factors, including adhesins, toxins, iron uptake systems (siderophores) and capsules, which are necessary for colonization and infection of different physiological compartments and often overlap [13]. Thus, some APEC strains are host independent and can cause diseases in hosts other than poultry, such as pigs and humans, that underline their zoonotic potential $[13,14]$.

Poultry and pigs carrying E. coli strains capable of causing extraintestinal infections and expressing the $3 \mathrm{MDRO}$ resistance phenotype introduce such bacteria into slaughterhouses. During subsequent processing, many process waters and wastewater arise that are contaminated by numerous bacteria, which mainly originate from livestock feces. This poses an elevated risk of colonization and infection of employees with occupational exposure to contaminated waters [15]. Furthermore, the introduction of clinically-relevant antibiotic-resistant bacteria into the food chain through cross-contaminations with polluted scalding water or process water from eviscerators cannot be ruled out [16]. Moreover, through inadequate wastewater treatment in in-house wastewater treatment plants (WWTPs), such bacteria could be discharged into the surface water and be further disseminated in the environment [17].

However, only limited data are available on the epidemiology of multidrug-resistant and extraintestinal pathogenic E. coli in German poultry and pig slaughterhouses. Thus, this study aims to characterize selected $E$. coli isolates expressing clinically relevant resistance phenotypes (3MDRO, ceftolozane-tazobactam (C/T) and/or ceftazidime-avibactam (CZA)), and which were allocated to the ExPEC groups regarding their antimicrobial resistance genes, population structure and virulence factors.

\section{Materials and Methods}

\subsection{Sampling Sites and Sample Preparation}

Collection and preparation of process waters and wastewater from two poultry and two pig slaughterhouses, as well as their in-house WWTPs, were conducted as previously described (Savin et al. 2020a, 2020b). Therefore, individual water samples (1 L) were collected using sterile Nalgene ${ }^{\circledR}$ wide mouth environmental sample bottles (Thermo Fisher Scientific, Waltham, MA, USA). Afterward, they were transported to the laboratory in a Styrofoam box cooled to $5 \pm 2{ }^{\circ} \mathrm{C}$, manually filtered using stomacher strainer bags with a 
tissue filter (pore size, $0.5 \mathrm{~mm}$; VWR, Radnor, PA, USA) and subjected to cultivation within $24 \mathrm{~h}$ after sampling. The investigated slaughterhouses were located in different federal states and were at least $100 \mathrm{~km}$ apart. Further information on selected slaughterhouses characteristics, sampling sites, number of samples taken at each sampling site and sampling dates is summarized in Tables S1-S3.

A total of 149 water samples were included in the present study. Briefly, 82 water samples originating from poultry slaughterhouses were taken from eight sampling sites: transport trucks $(n=5)$, transport crates $(n=10)$, stunning facilities $(n=10)$, scalders $(n=10)$, eviscerators $(n=10)$, aggregate wastewater from production facilities $(n=5)$ as well as influent ( $n=16)$ and effluent $(n=16)$ of the in-house WWTPs) [6]. Another set of 67 water samples from pig slaughterhouses was collected in the delivery (transport trucks, $n=10$; holding pens, $n=7$ ) and unclean areas (scalding and dehairing water, $n=10$; aggregate wastewater from production facilities, $n=10)$ as well as in the in-house WWTPs (influent $(n=15)$ and effluent $(n=15))$ [7].

\subsection{Isolation and Identification of Target E. coli}

Isolation of ESBL-producing E. coli was conducted using CHROMagar ESBL selective media (Mast Diagnostica, Reinfeld, Germany) as previously described [6,7]. Isolates were purified by streaking out individual colonies on Columbia Agar supplemented with 5\% sheep blood (ColSB, Mast Diagnostics, Reinfeld, Germany). Species identification was conducted using a MALDI-TOF MS employing a VITEK ${ }^{\circledR}$ mass spectrometer (bioMérieux, Marcy-1'Étoile, France) equipped with the Myla ${ }^{\mathrm{TM}}$ software.

\subsection{Antimicrobial Susceptibility Testing (AST) and Molecular Typing}

AST was performed by broth microdilution using commercial screening system Micronaut S MDR MRGN (MERLIN, Gesellschaft für mikrobiologische Diagnostika GmbH, Bornheim-Hersel, Germany) and applying clinical cut-off values (EUCAST, version 9.0) as previously described [6]. This screening system was chosen in order to assess the clinical relevance of isolates recovered from slaughterhouses for human medicine. Multidrugresistance phenotype (3MDRO) was defined as a combined resistance to piperacillin (PIP), cefotaxime (CTX) and/or ceftazidime (CAZ), and ciprofloxacin (CIP) [18]. The following antimicrobial agents were used in this study: temocillin, TEM; piperacillin-tazobactam, TZP; cefotaxime, CTX; ceftazidime, CAZ; ceftazidime-avibactam, CZA; ceftolozane-tazobactam, C/T; imipenem, IMP; meropenem, MEM; amikacin, AMK; ciprofloxacin, CIP; levofloxacin, LVX; sulfamethoxazole-trimethoprim, SXT; fosfomycin, FOF; colistin, CST.

For the determination of phylogenetic groups (A, B1, B2, C, D, E, F and clade I) and identification of extraintestinal pathogenic (ExPEC) strains, E. coli isolates were genotyped according to the method of Clermont and colleagues (2013) [19].

Allocation to ExPEC and uropathogenic E. coli (UPEC) pathotypes was done according to Johnson and colleagues (2003) [20] and Spurbeck and colleagues (2012) [21], respectively. The ExPEC pathotype was defined by the presence of $\geq 2$ virulence genes (VGs) coding for adhesins (papA/papC, sfa/foc, afa/dra), protectins (kpsM II) and/or iron uptake systems (iut $A$ ). The presence of $\geq 2$ VGs encoding iron uptake systems (chuA, fyuA), toxins (vat) and/or adhesins $\left(y f_{c} V\right)$ defined the UPEC pathotype.

\subsection{DNA Preparation, Whole-Genome Sequencing and Bioinformatics Analysis}

For paired-end, short-read whole-genome sequencing (WGS) on an Illumina NextSeq 500 device, DNA libraries prepared with the Nextera DNA Flex library preparation kit (Illumina ${ }^{\circledR}$, San Diego, CA, USA) according to the manufacturer's protocol were used. NextSeq sequencing was performed in $2 \times 151$ cycles with the Illumina ${ }^{\circledR}$ NextSeq $^{\mathrm{TM}}$ 500/550 Mid Output Kit v2.5 (300 cycles) (Illumina ${ }^{\circledR}$, San Diego, CA, USA) (Borowiak et al. 2017). Isolation of genomic DNA for $1 \mathrm{~mL}$ liquid cultures of bacteria was conducted using the PureLink Genomic DNA Preparation Mini Kit (Invitrogen GmbH, Darmstadt, Germany) recommended by the manufacturers. Illumina raw reads were trimmed and subjected to 
de novo assembling with the Aquamis pipeline (https:/ / gitlab.com/bfr_bioinformatics / AQUAMIS/, access date: September 2020) using fastp [22] for trimming and shovill (https:/ / github.com/tseemann/shovill, access date: September 2020) for genome assembly. The Aquamis pipeline further uses mash v 2.1 for reference search [23] and quast v 5.0.2 for assembly quality control [24]. In silico based characterization of genetic features was performed with the Bakcharak pipeline (https://gitlab.com/bfr_bioinformatics/bakcharak, access date: September 2020) implementing ABRicate (https://github.com/tseemann/ abricate, access date: September 2020) for antimicrobial/biocide resistance [25] and virulence factors detection [26]. Multi-locus sequence types (MLST) were predicted using mlst (https:/ / github.com/tseemann/mlst, access date: September 2020) [27].

\section{Results}

\subsection{Isolation and Selection of target E. coli}

In general, out of 376 recovered $E$. coli isolates ( $n=186$ poultry slaughterhouses; $n=190$ pig slaughterhouses), 71 isolates (18.9\%) were chosen for further analysis. The selection was based on at least one of the following criteria: (i) development of a 3MDRO resistance phenotype; (ii) resistance to the newly approved drug combinations ceftazidime-avibactam and/or ceftolozane-tazobactam, (iii) allocation to the ExPEC phylogroups B2/D/F.

Of the selected E. coli isolates, 35 were recovered from process waters and wastewater generated in poultry slaughterhouses during operation and cleaning of facilities: transport trucks $(n=2)$; transport cages $(n=3)$; stunning facilities $(n=7)$; scalding water $(n=2)$; eviscerators $(n=6)$; aggregate wastewater from production facilities $(n=3)$; influent inhouse WWTP $(n=5)$ and effluent in-house WWTP $(n=7)$. From process waters and wastewater accruing in pig slaughterhouses, $36 \mathrm{E}$. coli isolates were chosen: transport trucks $(n=4)$; holding pens $(n=3)$; scalding water $(n=3)$; production facilities $(n=7)$; influent biological WWTP $(n=9)$; influent chemical-physical WWTP $(n=4)$ and effluent biological WWTP $(n=6)$. Information on the isolation sites of individual isolates and their accession numbers are provided in Table S4.

\subsection{Phenotypic Antimicrobial Resistance}

Selected $E$. coli isolates ( $n=35$ poultry slaughterhouses; $n=36$ pig slaughterhouses) exhibited diverse resistance phenotypes, including resistance to highly and critically important antimicrobials for humans (Figure 1). Furthermore, they expressed high multidrugresistance rates. Of the isolates recovered from the poultry and pig slaughterhouses, $51.4 \%$ and $58.3 \%$, respectively, expressed combined resistance to piperacillin (PIP), cefotaxime (CTX) and/or ceftazidime (CAZ), and ciprofloxacin (CIP). The rates of resistance to combinations of $\beta$-lactam/ $\beta$-lactamase inhibitor (i.e., piperacillin-tazobactam, TZP; ceftazidime-avibactam, CZA; ceftolozane-tazobactam, C/T) was between $2.9 \%$ and $17.1 \%$, whereas the highest rates were observed for $\mathrm{C} / \mathrm{T}$. Furthermore, of the isolates recovered from the poultry and pig slaughterhouses, $17.1 \%$ and $19.4 \%$, respectively, expressed resistance to colistin. Noteworthy, all isolates were susceptible to carbapenems (imipenem, meropenem), amikacin and tigecycline.

\subsection{Characterization of Antimicrobial Resistance Genes}

In selected $E$. coli isolates from poultry slaughterhouses, 53 antimicrobial resistance genes (ARGs) belonging to 13 different classes were identified, whereas isolates from pig slaughterhouses represented a reservoir for 52 ARGs of 13 different classes. Of these, 39 ARGs were common for isolates from both poultry and pig slaughterhouses (Figure 2, Figure 3). 


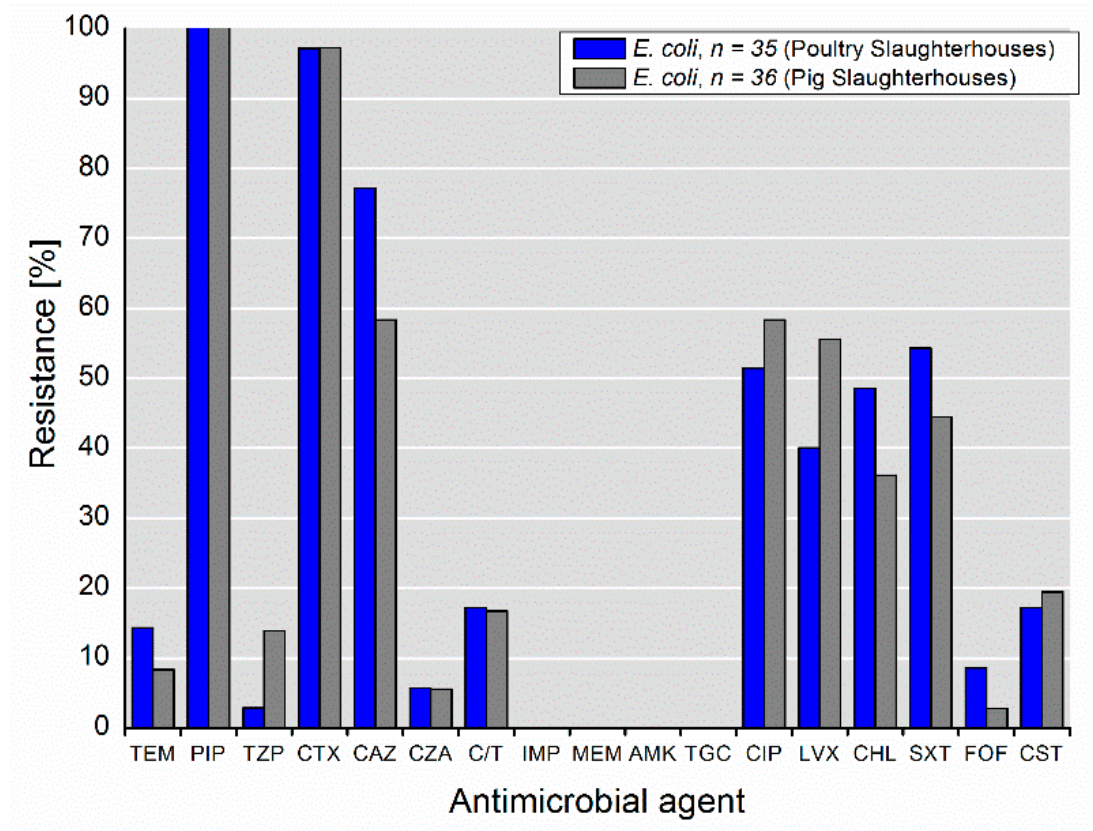

Figure 1. Phenotypical resistance to antimicrobial agents detected among selected $E$. coli isolates $(n=71)$ from wastewater and process water from poultry and pig slaughterhouses.

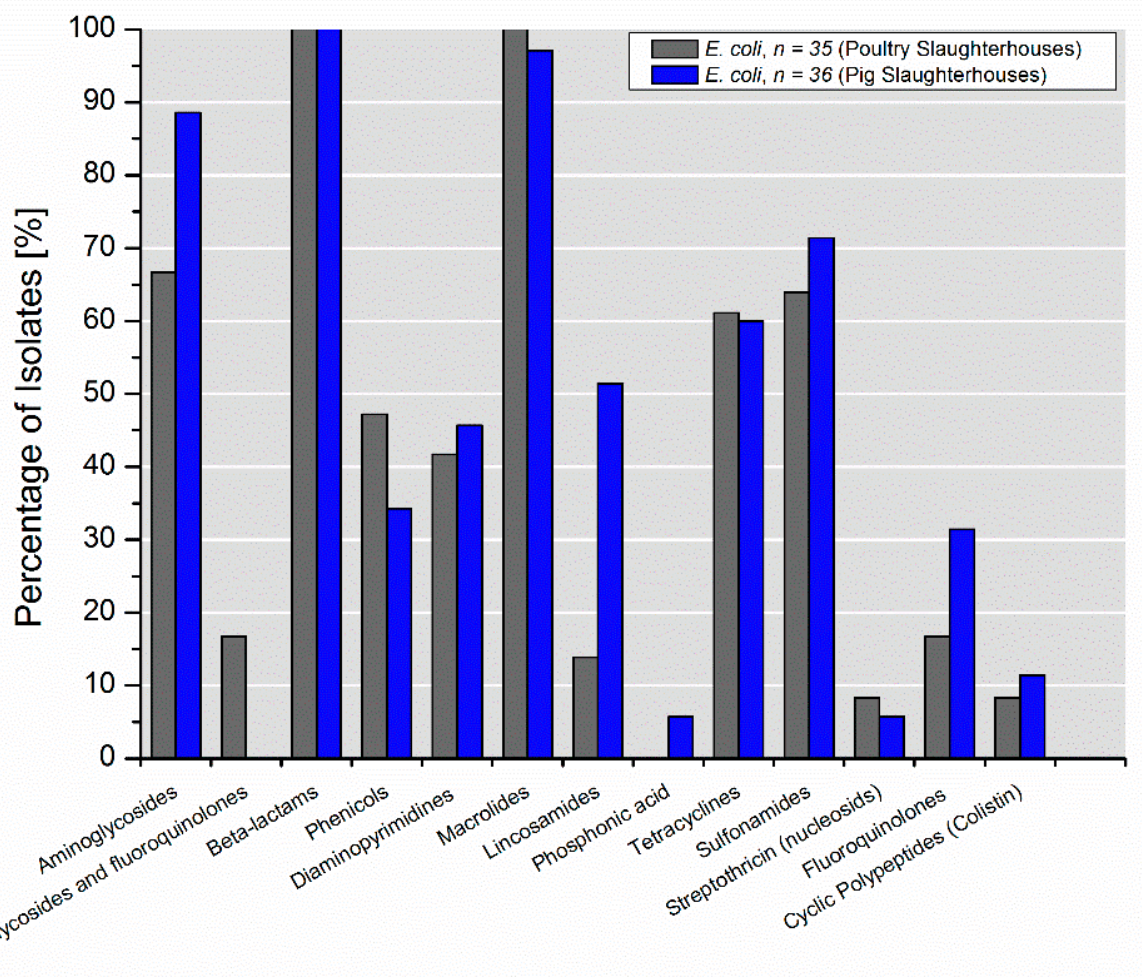

Figure 2. Percentage of selected E. coli isolates $(n=71)$ recovered from wastewater and process waters from poultry and pig slaughterhouses carrying genes mediating resistance to the specific classes of antimicrobials. 


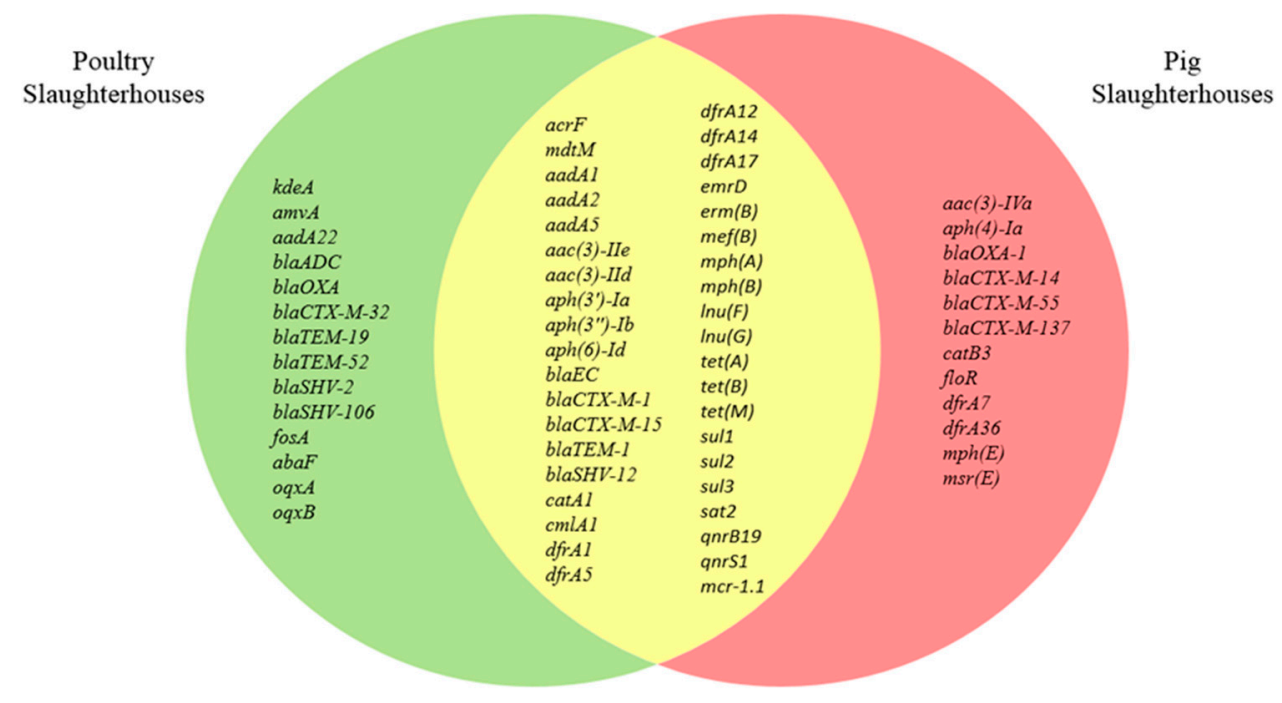

Figure 3. Antibiotic resistance genes identified in selected E. coli isolates $(n=71)$ from poultry and pig slaughterhouses.

Compared to the isolates from pig slaughterhouses, a higher abundance of ARGs coding for resistance to lincosamides $\ln u(F)$ and $\ln u(G)(51.4 \%$ vs. $13.9 \%)$ was detected in isolates from poultry slaughterhouses (Table 1). Interestingly, $16.7 \%(6 / 36)$ of the isolates from pig slaughterhouses harbored gene encoding resistance to aminoglycosides and fluoroquinolones $\left(a a c\left(6^{\prime}\right)-I b-c r 5\right)$. As expected, $\beta$-lactamases genes that belonged to the $b l a_{\mathrm{ADC}}, b l a_{\mathrm{OXA}}, b l_{\mathrm{EC}}, b l a_{\mathrm{CTX}-\mathrm{M}}, b l a_{\mathrm{TEM}}, b l a_{\mathrm{SHV}}$ families were detected in all selected isolates since selective media were used for their culturing. However, only five $\beta$-lactamases genes $\left(b l_{\mathrm{EC}}, b l a_{\mathrm{CTX}-\mathrm{M}-1}, b a_{\mathrm{CTX}-\mathrm{M}-15}, b l a_{\mathrm{TEM}-1}, b a_{\mathrm{SHV}-12}\right)$ of 16 identified ones were present in isolates from both poultry and pig slaughterhouses. Interestingly, bla-OXA-1 was detected in combination with extended-spectrum $\beta$-lactamases (ESBLs), such as bla $a_{\mathrm{CTX}-\mathrm{M}-1}$ and $b l a_{\mathrm{CTX}-\mathrm{M}-15}$ only in isolates from both pig slaughterhouses. Noteworthy, ARGs coding for resistance to macrolides was detected in all isolates with very few exceptions among isolates from pig slaughterhouses. They were mostly represented by emrD or combinations of $e m r D$ with $m p h(A)$ or $m p h(B)$. ARGs conferring resistance to phosphonic acid (fos $A, a b a F$ ) and streptothricin (sat2) were found only rarely with abundances $<10 \%$.

Table 1. Antibiotic resistance genes and their combinations detected in selected E. coli isolates recovered from wastewater and process waters from poultry and pig slaughterhouses.

\begin{tabular}{|c|c|c|c|c|}
\hline & \multicolumn{2}{|c|}{ Poultry Slaughterhouses, $n=35$} & \multicolumn{2}{|c|}{ Pig Slaughterhouses, $n=36$} \\
\hline & Genes & Percentage & Genes & Percentage \\
\hline \multirow{8}{*}{ Aminoglycosides } & $\operatorname{aad} A 1$, aad $A 2$ & 22.9 & $\operatorname{aad} A 1$ & 8.3 \\
\hline & $\operatorname{aad} A 2$ & 14.3 & aad $A 1$, aad $A 2$ & 8.3 \\
\hline & aadA1, aph(3")-Ib, aph(6)-Id & 11.4 & aac(3)-IIe, aph(3")-Ib, aph(6)-Id & 5.6 \\
\hline & aadA22 & 11.4 & $\begin{array}{c}\operatorname{aad} A 1, \operatorname{aad} A 2, \operatorname{aph}\left(3^{\prime \prime}\right)-I b \\
\operatorname{aph}(6)-I d\end{array}$ & 5.6 \\
\hline & $\operatorname{aac}(3)-I I e$, aadA1 & 5.7 & $\operatorname{aadA1}, \operatorname{aph}\left(3^{\prime \prime}\right)-I b, \operatorname{aph}(6)-I d$ & 5.6 \\
\hline & $\begin{array}{l}\text { aadA, aadA5, aph(3")-Ib, } \\
\text { aph(6)-Id }\end{array}$ & 5.7 & $\begin{array}{l}\text { aac(3)-IId, } \operatorname{aph}\left(3^{\prime \prime}\right)-I b, a p h\left(3^{\prime}\right)-I a, \\
\operatorname{aph}(6)-I d\end{array}$ & 2.8 \\
\hline & $\operatorname{aac}(3)-I I d$, aadA2 & 2.9 & $\operatorname{aac}(3)-I I e, \operatorname{aadA1}$ & 2.8 \\
\hline & $\begin{array}{l}\operatorname{aad} A 1, \operatorname{aad} A 2, \operatorname{aph}\left(3^{\prime \prime}\right)-I b \\
\operatorname{aph}\left(3^{\prime}\right)-I a, \operatorname{aph}(6)-I d\end{array}$ & 2.9 & $\operatorname{aac}(3)-I I e, \operatorname{aad} A 1, \operatorname{aph}\left(3^{\prime}\right)-I a$ & 2.8 \\
\hline
\end{tabular}


Table 1. Cont.

\begin{tabular}{|c|c|c|c|c|}
\hline & \multicolumn{2}{|c|}{ Poultry Slaughterhouses, $n=35$} & \multicolumn{2}{|c|}{ Pig Slaughterhouses, $n=36$} \\
\hline \multirow{11}{*}{ Aminoglycosides } & Genes & Percentage & Genes & Percentage \\
\hline & $\begin{array}{c}\text { aadA1, aadA5, aph(3")-Ib, } \\
\text { aph(6)-Id }\end{array}$ & 2.9 & $\begin{array}{c}\text { aac(3)-IIe, aadA5, aph(3")-Ib, } \\
\text { aph(6)-Id }\end{array}$ & 2.8 \\
\hline & $\operatorname{aad} A 2, \operatorname{aph}\left(3^{\prime \prime}\right)-I b, \operatorname{aph}(6)-I d$ & 2.9 & aac(3)-IIe, aph(3")-Ib, aph(6)-Id & 2.8 \\
\hline & $\begin{array}{c}\text { aad } A 22, \\
\operatorname{aph}\left(3^{\prime \prime}\right)-I b ; a p h\left(3^{\prime}\right)-I a \\
\operatorname{aph}(6)-I d\end{array}$ & 2.9 & $\begin{array}{l}\operatorname{aac}(3)-I V a, \operatorname{aadA1}, \operatorname{aph}\left(3^{\prime \prime}\right)-I b \\
\operatorname{aph}\left(3^{\prime}\right)-I a, \operatorname{aph}(4)-I a, \operatorname{aph}(6)-I d\end{array}$ & 2.8 \\
\hline & aadA5, aph(3")-Ib, aph(6)-Id & 2.9 & $\operatorname{aad} A 1$, aad $A 5$ & 2.8 \\
\hline & & & $\begin{array}{l}\operatorname{aad} A 1, \text { aad } A 5, \operatorname{aph}\left(3^{\prime \prime}\right)-I b, \\
\operatorname{aph}\left(3^{\prime}\right)-I a, \operatorname{aph}(6)-I d\end{array}$ & 2.8 \\
\hline & & & $\operatorname{aad} A 5$ & 2.8 \\
\hline & & & aadA5, aph(3")-Ib, aph(6)-Id & 2.8 \\
\hline & & & $\operatorname{aph}\left(3^{\prime \prime}\right)-I b, a p h\left(3^{\prime}\right)-I a, a p h(6)-I d$ & 2.8 \\
\hline & & & $\operatorname{aph}\left(3^{\prime \prime}\right)-I b, a p h(6)-I d$ & 2.8 \\
\hline & Overall & 88.6 & & 66.7 \\
\hline \multirow{2}{*}{$\begin{array}{l}\text { Aminoglycosides and } \\
\text { Fluoroquinolones }\end{array}$} & & & $a a c\left(6^{\prime}\right)-I b-c r 5$ & 16.7 \\
\hline & Overall & & & 16.7 \\
\hline \multirow{13}{*}{$\beta$-lactams } & $b l a_{\mathrm{EC}}, b l a_{\mathrm{SHV}-12}$ & 20.0 & $b l a_{\mathrm{CTX}-\mathrm{M}-1}, b l a_{\mathrm{EC}}$ & 25.0 \\
\hline & $b l a_{\mathrm{EC}}, b l a_{\mathrm{TEM}-52}$ & 17.1 & $b l a_{\mathrm{CTX}-\mathrm{M}-1}, b l a_{\mathrm{EC}}, b l a_{\mathrm{TEM}-1}$ & 22.2 \\
\hline & $b l a_{\mathrm{EC}}, b l a_{\mathrm{TEM}-1}$ & 11.4 & $b l a_{\mathrm{CTX}-\mathrm{M}-15}, b l a_{\mathrm{EC}}, b l a_{\mathrm{OXA}-1}$ & 13.9 \\
\hline & $b l a_{\mathrm{CTX}-\mathrm{M}-1}, b l a_{\mathrm{EC}}, b l a_{\mathrm{TEM}-1}$ & 8.6 & $b l a_{\mathrm{CTX}-\mathrm{M}-55}, b l a_{\mathrm{EC}}$ & 8.3 \\
\hline & $b l a_{\mathrm{CTX}-\mathrm{M}-32}, b l a_{\mathrm{EC}}, b l a_{\mathrm{TEM}-1}$ & 8.6 & $b l a_{\mathrm{EC}}, b l a_{\mathrm{OXA}-1}, b l a_{\mathrm{TEM}-1}$ & 8.3 \\
\hline & $b l a_{\mathrm{EC}}, b l a_{\mathrm{SHV}-12}, b l a_{\mathrm{TEM}-1}$ & 8.6 & $b l a_{\mathrm{CTX}-\mathrm{M}-15}, b l a_{\mathrm{EC}}, b l a_{\mathrm{TEM}-1}$ & 5.6 \\
\hline & $b l a_{\mathrm{CTX}-\mathrm{M}-1}, b l a_{\mathrm{EC}}$ & 5.7 & $b l a_{\mathrm{EC}}, b l a_{\mathrm{SHV}-12}$ & 5.6 \\
\hline & $b l a_{\mathrm{CTX}-\mathrm{M}-15}, b l a_{\mathrm{EC}}, b l a_{\mathrm{TEM}-1}$ & 5.7 & $b l a_{\mathrm{CTX}-\mathrm{M}-1}, b l a_{\mathrm{EC}}, b l a_{\mathrm{OXA}-1}$ & 2.8 \\
\hline & $b l a_{\mathrm{EC}}, b l a_{\mathrm{SHV}-2}, b l a_{\mathrm{TEM}-1}$ & 5.7 & $b l a_{\mathrm{CTX}-\mathrm{M}-137}, b l a_{\mathrm{EC}}, b l a_{\mathrm{TEM}-1}$ & 2.8 \\
\hline & $b l a_{\mathrm{ADC}}, b l a_{\mathrm{OXA}}$ & 2.9 & $b l a_{\mathrm{CTX}-\mathrm{M}-14}, b l a_{\mathrm{EC}}, b l a_{\mathrm{TEM}-1}$ & 2.8 \\
\hline & 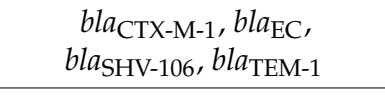 & 2.9 & $\begin{array}{c}b l a_{\mathrm{CTX}-\mathrm{M}-15}, b l a_{\mathrm{EC}}, b l a_{\mathrm{OXA}-1}, \\
\text { bla } \\
\text { TEM-1 }\end{array}$ & 2.8 \\
\hline & $b l a_{\mathrm{EC}}, b l a_{\mathrm{TEM}-1}, b l a_{\mathrm{TEM}-19}$ & 2.9 & & \\
\hline & Overall & 100 & & 100 \\
\hline \multirow{7}{*}{ Phenicols } & cmlA1 & 17.1 & catB3 & 13.9 \\
\hline & cat A1 & 11.4 & floR & 11.1 \\
\hline & cat $A 1, \mathrm{cmlA1}$ & 5.7 & cat $A 1$, floR & 8.3 \\
\hline & & & cmlA1, floR & 8.3 \\
\hline & & & catB3, cmlA1 & 2.8 \\
\hline & & & cmlA1 & 2.8 \\
\hline & Overall & 34.3 & & 47.2 \\
\hline \multirow{4}{*}{ Diaminopyrimidines } & $d f r A 1$ & 14.3 & $d f r A 17$ & 13.9 \\
\hline & dfrA12 & 8.6 & $d f r A 12, d f r A 36$ & 8.3 \\
\hline & $d f r A 17$ & 5.7 & $d f r A 1$ & 2.8 \\
\hline & $d f r A 1, d f r A 12$ & 2.9 & $d f r A 1, d f r A 17$ & 2.8 \\
\hline
\end{tabular}


Table 1. Cont.

\begin{tabular}{|c|c|c|c|c|}
\hline & \multicolumn{2}{|c|}{ Poultry Slaughterhouses, $n=35$} & \multicolumn{2}{|c|}{ Pig Slaughterhouses, $n=36$} \\
\hline \multirow{7}{*}{ Diaminopyrimidines } & Genes & Percentage & Genes & Percentage \\
\hline & $d f r A 1, d f r A 14$ & 2.9 & dfrA12 & 2.8 \\
\hline & $d f r A 1, d f r A 17$ & 2.9 & $d f r A 12, d f r A 5$ & 2.8 \\
\hline & dfrA12, dfrA17 & 2.9 & $d f r A 14$ & 2.8 \\
\hline & dfrA14 & 2.9 & $d f r A 17, d f r A 7$ & 2.8 \\
\hline & dfrA5 & 2.9 & dfrA5 & 2.8 \\
\hline & Overall & 45.7 & & 41.7 \\
\hline \multirow{6}{*}{ Macrolides } & emrD & 77.1 & emrD & 61.1 \\
\hline & $e m r D, m p h(B)$ & 8.6 & $\operatorname{emrD}, \operatorname{mph}(A)$ & 30.6 \\
\hline & $\operatorname{emrD}, \operatorname{mph}(A)$ & 5.7 & $\operatorname{emrD}, \operatorname{erm}(B)$ & 2.8 \\
\hline & $e m r D, \operatorname{erm}(B)$ & 2.9 & $\operatorname{emrD}, m p h(A), m p h(B)$ & 2.8 \\
\hline & $\operatorname{emr} D, \operatorname{mef}(B)$ & 2.9 & emrD, mphE, mef(B),msrE & 2.8 \\
\hline & Overall & 97.1 & & 100 \\
\hline \multirow{3}{*}{ Lincosamides } & $\ln u(F)$ & 48.6 & $\ln u(G)$ & 11.1 \\
\hline & $\ln u(F), \ln u(G)$ & 2.9 & $\ln u(F)$ & 2.8 \\
\hline & Overall & 51.4 & & 13.9 \\
\hline \multirow{3}{*}{$\begin{array}{l}\text { Phosphonic Acid } \\
\text { (Fosfomycin) }\end{array}$} & fos $A$ & 2.9 & & \\
\hline & $a b a F$ & 2.9 & & \\
\hline & Overall & 5.8 & & \\
\hline \multirow{5}{*}{ Tetracyclines } & $\operatorname{tet}(A)$ & 48.6 & $\operatorname{tet}(A)$ & 36.1 \\
\hline & $\operatorname{tet}(B)$ & 8.6 & $\operatorname{tet}(B)$ & 13.9 \\
\hline & $\operatorname{tet}(A), \operatorname{tet}(M)$ & 2.9 & $\operatorname{tet}(A), \operatorname{tet}(M)$ & 8.3 \\
\hline & & & $\operatorname{tet}(A), \operatorname{tet}(B)$ & 2.8 \\
\hline & Overall & 60.0 & & 61.1 \\
\hline \multirow{7}{*}{ Sulfonamides } & sul2 & 28.6 & sul2 & 25.0 \\
\hline & sul1, sul2 & 14.3 & sul1, sul2, sul3 & 11.1 \\
\hline & sul3 & 14.3 & sul1 & 8.3 \\
\hline & sul1 & 5.7 & sul1, sul2 & 8.3 \\
\hline & sul2, sul3 & 5.7 & sul3 & 8.3 \\
\hline & sul1, sul3 & 2.9 & sul2, sul3 & 2.8 \\
\hline & Overall & 71.4 & & 63.9 \\
\hline \multirow{2}{*}{$\begin{array}{l}\text { Streptothricin } \\
\text { (Nucleosides) }\end{array}$} & sat2 & 5.7 & sat2 & 8.3 \\
\hline & Overall & 5.7 & & 8.3 \\
\hline \multirow{4}{*}{ Fluoroquinolones } & qnrS1 & 17.1 & qnrS1 & 11.1 \\
\hline & $q n r B 19$ & 11.4 & $q n r B 19$ & 5.6 \\
\hline & oq $x A$, oq $x B$ & 2.9 & & \\
\hline & Overall & 31.4 & & 16.7 \\
\hline \multirow{2}{*}{$\begin{array}{l}\text { Cyclic Polypeptides } \\
\text { (Colistin) }\end{array}$} & $m c r-1.1$ & 11.4 & $m c r-1.1$ & 8.3 \\
\hline & Overall & 11.4 & & 8.3 \\
\hline
\end{tabular}


In general, there was a good concordance between the resistance phenotypes and the resistance genes identified by WGS for isolates from both poultry and pig slaughterhouses.

\subsection{Distribution of Phylogenetic Groups and MLST Sequence Types}

Selected E. coli isolates from poultry slaughterhouses $(n=35)$ mainly belonged to the group B1 (34.3\%), followed by F (17.1\%), E (14.3\%), A and D (each $11.4 \%), \mathrm{C}(8.6 \%)$ as well as B2 $(2.9 \%)$. Most of the isolates from pig slaughterhouses $(n=36)$ were also assigned to B1 group (41.7\%), followed by A (25.0\%), C (22.2\%), B2 (5.6\%) and D (5.6\%).

MLST revealed a high genetic diversity of selected E. coli isolates. Overall, 66 isolates were assigned to 42 distinct previously described sequence types (STs), whereas five isolates exhibited novel STs (Table 2). Isolates from poultry slaughterhouses belonged to 25 different STs, whereas isolates from pig slaughterhouses exhibited 22 STs. Interestingly, five STs (ST10, ST58, ST101, ST117, ST224) were common for isolates from poultry and pig slaughterhouses.

Table 2. Multi-locus sequence types (MLST) distribution of selected E. coli isolates recovered from poultry and pig slaughterhouses.

E. coli, $n=35$

Poultry Slaughterhouses
E. coli, $n=36$

Pig Slaughterhouses

\begin{tabular}{cccccc}
\hline $\begin{array}{c}\text { Sequence } \\
\text { Type }\end{array}$ & $n$ & $\%$ & $\begin{array}{c}\text { Sequence } \\
\text { Type }\end{array}$ & $n$ & $\%$ \\
\hline ST117 & 3 & 8.6 & ST410 & 5 & 13.9 \\
\hline ST10 & 2 & 5.7 & ST10 & 4 & 11.1 \\
\hline ST224 & 2 & 5.7 & ST359 & 3 & 8.3 \\
\hline ST533 & 2 & 5.7 & ST744 & 3 & 8.3 \\
\hline ST648 & 2 & 5.7 & ST58 & 2 & 5.6 \\
\hline ST1011 & 2 & 5.7 & ST88 & 2 & 5.6 \\
\hline ST1730 & 2 & 5.7 & ST117 & 2 & 5.6 \\
\hline ST3995 & 2 & 5.7 & ST101 & 1 & 2.8 \\
\hline ST4994 & 2 & 5.7 & ST131 & 1 & 2.8 \\
\hline ST34 & 1 & 2.9 & ST156 & 1 & 2.8 \\
\hline ST58 & 1 & 2.9 & ST167 & 1 & 2.8 \\
\hline ST101 & 1 & 2.9 & ST224 & 1 & 2.8 \\
\hline ST135 & 1 & 2.9 & ST398 & 1 & 2.8 \\
\hline ST155 & 1 & 2.9 & ST542 & 1 & 2.8 \\
\hline ST162 & 1 & 2.9 & ST617 & 1 & 2.8 \\
\hline ST297 & 1 & 2.9 & ST641 & 1 & 2.8 \\
\hline ST361 & 1 & 2.9 & ST1170 & 1 & 2.8 \\
\hline ST457 & 1 & 2.9 & ST1284 & 1 & 2.8 \\
\hline ST515 & 1 & 2.9 & ST1431 & 1 & 2.8 \\
\hline ST711 & 1 & 2.9 & ST3595 & 1 & 2.8 \\
\hline ST1485 & 1 & 2.9 & & & \\
\hline ST6617 & 1 & 2.9 & & & 5.6 \\
\hline unknown STs & 3 & 8.6 & unknown STs & 2 & \\
\hline & & & & & \\
\hline
\end{tabular}

\subsection{Characterization of Virulence Genes}

The virulence genes of the analyzed E. coli isolates are summarized in Table 3. In general, genes coding for virulence factors, such as adhesins, toxins, siderophores and capsules, 
were detected. Interestingly, almost all isolates carried fimH that codes for the adhesin on type 1 pili. Of note, $40.0 \%$ of isolates from poultry slaughterhouses $(14 / 35)$ carried virulence gene ast $A$ encoding the enteroaggregative $E$. coli heat-stable enterotoxin (EAST1), whereas this virulence genotype was less present among isolates from pig slaughterhouses $(16.7 \%, 6 / 36)$. Nevertheless, one isolate recovered from the wastewater used for cleaning pig transport trucks carried virulence determinants coding for $\alpha$-hemolysin $(h l y D)$ and cytotoxic necrotizing factor ( $c n f 1$ ). Furthermore, a high percentage of isolates was positive for different siderophores, such as aerobactin (iutA), salmochelin (iroN) and yersiniabactin $(f y u A)$, whereas aerobactin was the most prevalent one. Noteworthy, the percentage of isolates carrying genes for group 2 capsule ( $k p s M ~ I I)$ was higher among isolates from poultry slaughterhouses $(22.9 \%, 8 / 35)$ compared to those from pig slaughterhouses $(5.6 \%, 2 / 36)$.

Table 3. Virulence factors detected in selected E. coli isolates recovered from wastewater and process waters from poultry and pig slaughterhouses.

\begin{tabular}{|c|c|c|c|}
\hline & & $\begin{array}{c}\text { E. coli }(n=35), \% \\
\text { Poultry Slaughterhouses }\end{array}$ & $\begin{array}{c}\text { E. coli }(n=36), \% \\
\text { Pig Slaughterhouses }\end{array}$ \\
\hline \multicolumn{4}{|c|}{ Adhesins } \\
\hline $\mathrm{fimH}$ & Type 1 fimbriae & 94.3 & 91.7 \\
\hline papC & Genes of P fimbriae operon & 5.7 & 11.1 \\
\hline papEFG & Genes of P fimbriae operon & 5.7 & 8.3 \\
\hline$s f a / f o c$ & S or F1C fimbriae & 5.7 & 11.1 \\
\hline focG & F1C fimbriae adhesin & 0 & 0 \\
\hline iha & Adhesin siderophore & 0 & 0 \\
\hline F10 papA & P fimbriae subunit variant & 0 & 0 \\
\hline tsh & Temperature sensitive hemagglutinin & 0 & 0 \\
\hline hra & Heat-resistant agglutinin & 0 & 0 \\
\hline$a f a / d r a B C$ & Dr-binding adhesins & 0 & 0 \\
\hline \multicolumn{4}{|c|}{ Toxins } \\
\hline ast $A$ & Enteroaggregative E. coli toxin & 40.0 & 16.7 \\
\hline vat & Vacuolating toxin & 8.6 & 5.6 \\
\hline pic & Serine protease & 8.6 & 2.8 \\
\hline$h l y D$ & Alpha-hemolysin & 0 & 2.8 \\
\hline cnf1 & Cytotoxic necrotizing factor & 0 & 2.8 \\
\hline sat & Secreted autotransporter toxin & 0 & 0 \\
\hline \multicolumn{4}{|c|}{ Siderophores } \\
\hline iutA & Aerobactin receptor & 51.4 & 58.3 \\
\hline iroN & Salmochelin receptor & 48.6 & 30.6 \\
\hline fyuA & Yersiniabactin receptor & 37.1 & 27.8 \\
\hline ireA & Siderophore receptor & 0 & 0 \\
\hline \multicolumn{4}{|c|}{ Capsule } \\
\hline kpsM II & kpsM II group 2 capsule & 22.9 & 5.6 \\
\hline K1 & K1 group 2 capsule variants & 0 & 0 \\
\hline K2 & $\mathrm{K} 2$ group 2 capsule variants & 0 & 0 \\
\hline K5 & K5 group 2 capsule variants & 0 & 0 \\
\hline kpsMT III & Group 3 capsule & 0 & 0 \\
\hline
\end{tabular}


Of the isolates from poultry slaughterhouses, 17.1\% (6/35) were assigned to ExPEC pathotype (iutA, kpsM II), isolated from scalding water, eviscerators and aggregate wastewater from production facilities. Interestingly, they mostly belonged to phylogroup F (3/6), followed by E (2/6) and B2 (1/6). Among the isolates from pig slaughterhouses, the abundance of ExPEC pathotype was lower. Of the selected E. coli isolates, 5.6\% (2/36) belonged to ExPEC (phylogroup B2) carrying sfa (S fimbriae), kpsM II and iutA, kpsM II, respectively. They were isolated from wastewater used for cleaning of transport trucks and influent of biological WWTP. One isolate (D, ST117) recovered from the effluent of in-house WWTP of a poultry slaughterhouse $(2.8 \%)$ carried fyuA and vat (vacuolating toxin), which define UPEC (uropathogenic E. coli) pathotype. UPEC (B2, ST1170) was also detected in wastewater used for cleaning pig transport trucks $(2.8 \%, 1 / 36)$.

\subsection{Heavy Metal and Biocide Resistance}

The occurrence of heavy metal resistance genes in the analyzed E. coli isolates is shown in Table 4. In general, percentages of isolates carrying determinants conferring resistance to heavy metals were higher among isolates from pig slaughterhouses. They exhibited higher rates of resistance to copper, copper/silver, mercury, and silver. All isolates, but one from poultry slaughterhouses, carried genes conferring resistance to arsenic.

Table 4. Heavy metal resistance genes detected in E. coli isolates recovered from wastewater and process waters from poultry and pig slaughterhouses.

\begin{tabular}{ccc}
\hline Metal & $\begin{array}{c}E . \text { coli }(\boldsymbol{n}=\mathbf{3 5}), \boldsymbol{\%} \\
\text { Poultry Slaughterhouses }\end{array}$ & $\begin{array}{c}\text { E.coli }(\boldsymbol{n}=\mathbf{3 6}), \mathbf{\%} \\
\text { Pig Slaughterhouses }\end{array}$ \\
\hline Arsenic & 97.1 & 100.0 \\
\hline Copper & 5.7 & 13.9 \\
\hline Copper/silver & 5.7 & 27.8 \\
\hline Mercury & 25.7 & 38.9 \\
\hline Silver & 5.7 & 27.8 \\
\hline Tellurium & 2.9 & 2.8 \\
\hline Nickel & 2.9 & 0 \\
\hline
\end{tabular}

Of the isolates from poultry slaughterhouses, $42.9 \%(15 / 35)$ carried genes (emrE, $\operatorname{sug} E(c), m d f A, y d g E / y d g F, q a c$ and $\operatorname{sugE}(p))$ conferring resistance to biocides, such as quaternary ammonium compounds (QACs). Among isolates from pig slaughterhouses, its abundance was lower at 33.3\% (12/36).

\section{Discussion}

The study provides evidence on the diversity of antimicrobial resistance, genetic lineages, virulence factors of MDR, and extraintestinal pathogenic E. coli from process waters and wastewater isolated from German poultry and pig slaughterhouses.

Although aminoglycosides were not used for selective isolation of the investigated target bacteria, ARGs coding for their resistance was detected in almost all isolates, which is in contrast to results from routine resistance monitoring in Germany [28,29]. Aminoglycosides are veterinary critically important antimicrobials (VCIA), with specifically neomycin, dihydrostreptomycin and spectinomycin being very important for the treatment of septicemia as well as digestive, respiratory and urinary diseases in livestock [30]. In the human sector, aminoglycosides have been assigned to critically important antimicrobials (CIAs) due to their relevance for treating MDR Gram-negative bacteria [31]. Especially gentamicin, amikacin and tobramycin are of high importance as they are used in combination with $\beta$-lactams against emerging MDR Acinetobacter, Pseudomonas and Enterobacter species [32].

Although the general use of antimicrobials has decreased in recent years, consumption of aminoglycosides in the German veterinary sector has slightly increased [1]. Interest- 
ingly, although the highest amount of aminoglycosides was used in fattening chickens in Germany, the abundance of ARGs coding for aminoglycoside resistance in isolates from pig slaughterhouses was comparably high as in poultry isolates. This might be due to co-selection through other antimicrobials that are often used in pigs, e.g., macrolides, lincosamides and tetracyclines [1] or linked with our selective isolation procedure. However, the occurrence of $a a c\left(6^{\prime}\right)-\mathrm{Ib}-\mathrm{cr} 5$ in isolates from pig slaughterhouses is worrying, as the AAC (6')-Ib-cr5 enzyme confers reduced susceptibility to quinolones and amikacin [33] and is increasingly reported in human isolates of the genus Acinetobacter as well as the Pseudomonadaceae family. Both genera comprise species assigned to the ESKAPE bacteria, which cause the majority of hospital infections with antibiotic-resistant bacteria in the European Union (EU) and USA [34,35].

All isolates harbouring $a a c\left(6^{\prime}\right)-I b-c r 5$ also carried $b l a_{\mathrm{OXA}-1}$. This finding is in line with Livermore and colleagues (2019) [9], who found that resistance to piperacillin-tazobactam is often associated with $b l a_{\mathrm{OXA}-1}$ that encodes a penicillinase with weak affinity for inhibitors such as tazobactam or clavulanate and is commonly associated with co-carriage of aac ( $\left.6^{\prime}\right)$ $\mathrm{Ib}$-cr. Since all piperacillin-tazobactam-resistant $E$. coli carried $b l_{\mathrm{TEM}-1}$, its hyperproduction might be linked to this phenotype $[8,36]$.

Interestingly, E. coli isolates carrying aac (6')-Ib-cr 5 and $b l a_{\mathrm{OXA}-1}$ belong to sequence type ST410 reported as an extraintestinal pathogen worldwide, and dissemination was described between humans companions animals, wildlife, livestock, and the environment. Furthermore, this sequence type was also associated with the emergence and/or co-occurrence of carbapenemase genes like $b l a_{\mathrm{NDM}-5}$ and/or $b l a_{\mathrm{OXA}-181}$ in human isolates [37]. Fortunately, the isolates of this study, as well as those described in other animal populations, still

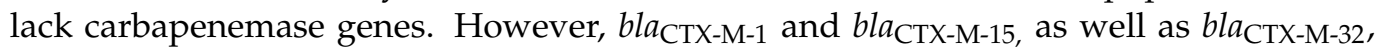
$b l a_{\mathrm{CTX}-\mathrm{M}-14}$ and $b l a_{\mathrm{CTX}-\mathrm{M}-55}$ that are often associated with ESBL-producing isolates from hospitals and ambulatory patients in Germany, were detected in isolates from both poultry and pig slaughterhouses [38].

Macrolides belong to the "highest priority critically important antimicrobials" (HPCIA) in human medicine. However, besides tetracyclines and penicillins, they belong to the most commonly used antimicrobials in fattening pigs in Germany [39]. The occurrence of resistance genes in almost all tested isolates is worrying since it is not excluded that the corresponding genes are located on the same plasmid as ARGs coding for resistance to $\beta$ lactam and aminoglycosides. Thus, macrolides in veterinary medicine may contribute to the co-selection and spread of resistance to CIAs for humans. Furthermore, erm genes could be transferred to Gram-positive pathogens (e.g., enterococci, streptococci, staphylococci) and result in $\mathrm{MLS}_{\mathrm{B}}$ (macrolide, lincosamide, streptogramin B) cross-resistance [40], compromising the efficacy of HPCIA for humans such as erythromycin macrolides and clindamycin.

As a notifiable number of isolates carried QAC genes, the use of QACs in the food industry, in particular in slaughterhouses, may provide additional selection pressure for clinically relevant $E$. coli with acquired resistance to other antimicrobial classes [41].

It is worrying that most detected STs in our study have also been reported in human infections worldwide $[11,38]$. ST10, ST58, ST88, ST117, ST131, ST167, ST410, ST617 and ST648 belong to the most widely disseminated extraintestinal pathogenic strains worldwide, causing infections of the bloodstream, urinary (UTI) and respiratory tracts as well as meningitis and necrotizing enterocolitis [11]. Furthermore, clones of ST34, ST101, ST155, ST361, ST744, ST1011, ST1170, ST1284 and ST1431 have been isolated in Germany from nosocomial and ambulant (UTIs) infections [38]. Of note, these clones were detected at all tested sampling sites along the slaughtering process, including scalding water (ST10, ST58, ST361) and the effluents of in-house WWTPs (ST10, ST117, ST410, ST648). E. coli allocated to STs detected in scalding water (ST10, ST58, ST361) have also been reported in chicken meat in different countries worldwide [42-44]. This emphasizes the potential role of polluted process waters for cross-contamination of carcasses and raw meat. Furthermore, our results show that in-house WWTPs of poultry and pig slaughterhouses may also play a relevant role in disseminating E. coli with zoonotic potential into the environment, 
including surface waters. E. coli ST10, ST117, ST410, ST648 have already been detected in surface waters in different European countries such as the Netherlands [45], Norway [46] and Switzerland [47], but it has to be mentioned that the sources of this contamination may have had multiple origins.

It is important that a significant percentage of $E$. coli isolates, especially from poultry slaughterhouses, belonged to the ExPEC pathotype that is known for its potential to cause human disease [48]. Such isolates were detected in scalding water and process water from eviscerators. As a consequence, cross-contamination of carcasses and their introduction into the food chain cannot be ruled out. Escherichia coli exhibiting an ExPEC pathotype have already been detected in a variety of different food products, including retail poultry meat $[14,49,50]$ and pork [51]. Some ExPEC isolates from animals have been shown to possess similar virulence gene profiles as human-associated ExPEC [52]. The mannose-binding type 1 pilus tip protein FimH plays a role in the invasion of ExPEC strains and their translocation from the intestine that can cause gut-derived bacteremia and sepsis [53]. Furthermore, FimH plays a vital role in lower UTI and kidney infections [54]. Ast $A$ that was detected in a notifiable number of recovered isolates, especially from poultry slaughterhouses, encodes the enteroaggregative E. coli heat-stable enterotoxin and might confer the ability to produce diarrhea [55]. Such isolates have already been associated with a waterborne outbreak of diarrhea [56]. Siderophores enable the acquisition and use of essential iron $\left(\mathrm{Fe}^{2+} / \mathrm{Fe}^{3+}\right)$ by bacteria and are essential for survival in the host [57]. KpsM II encodes a group 2 capsule known as a protection factor against phagocytosis [57]. Given that requirements for colonization of different physiological compartments often overlap, the incoming ExPEC isolates might be able to establish residence in the intestinal tract of the new host and cause disease afterward [58]. Because of the potential of these isolates to cause UTI in humans, these infections have been referred to as foodborne UTI [52].

Of special concern is the occurrence of UPEC strains in effluents of in-house WWTPs of poultry slaughterhouses since UPEC are the major cause of UTIs, accounting for 75\% of infection cases [59]. Zhi and colleagues (2020) suggested that UPEC of human origin appear to be specifically adapted to survive wastewater treatment processes such as chlorination, UV irradiation and activated sludge [60]. This elevates the concerns for public health since inadequate treatment of wastewater contributes to the establishment and persistence of environmental reservoirs of UPEC, facilitating their circulation among different populations.

Of particular importance is the high abundance of genes conferring arsenic resistance. Zhang and colleagues (2020) reported on the shift of antibiotic resistance genes (ARGs) and mobile genetic elements in bacteria from different surface waters (i.e., rivers, lakes, and reservoirs) due to arsenic pollution [61]. Furthermore, resistances to certain antimicrobials (e.g., enrofloxacin) are reported to be co-selected by other heavy metals, including copper, zinc, mercury, silver and nickel $[62,63]$. Heavy metals can reach high concentrations in different environmental settings, including agricultural production, and remain stable for prolonged periods of time [64], playing a notifiable role in the spread and proliferation of ARGs.

\section{Conclusions}

Occurrence of ESBL-producing, MDR E. coli with ExPEC, and UPEC pathotypes in process waters along poultry and pig slaughtering chains pose an elevated risk of employees' exposure to contaminated waters. The resistance patterns of the isolates among poultry or pig slaughterhouses were only slightly different. However, there were some considerable differences between isolates from the individual poultry and pig slaughterhouses, e.g., higher resistance rates to CIP, LVX and TZP among poultry isolates compared to those recovered from pig slaughterhouses. Furthermore, such clones of clinical relevance may be introduced into the food chain through cross-contamination of carcasses during scalding. Moreover, the inadequate treatment of the polluted wastewater in in-house WWTPs of poultry and pig slaughterhouses may result in disseminating antibiotic-resistant clinically 
relevant bacteria into the environment, e.g., receiving water bodies. Consequently, a contribution of these process waters, similarly to all effluents of WWTPs to a possible spread of bacteria into the general population, cannot be ruled out.

Supplementary Materials: The following are available online at https:/ / www.mdpi.com/article/10 $.3390 /$ microorganisms $9040698 /$ s1, Table S1: Selected characteristics of the investigated slaughterhouses; Table S2: Number of samples taken at each sampling point in poultry slaughterhouses; Table S3: Number of samples taken at each sampling point in pig slaughterhouses; Table S4: Isolation sites of the individual isolates and their accession numbers.

Author Contributions: Conceptualization, M.S. and J.A.H.; data curation, M.S.; funding acquisition, M.S., J.K. and J.A.H.; investigation, M.S., G.B., E.S. and J.A.H.; methodology, M.S., S.S. and J.A.H.; project administration, M.S. and J.A.H.; writing-original draft, M.S.; writing-review and editing, G.B., J.K., R.M.S., E.S., A.K. and J.A.H. All authors have read and agreed to the published version of the manuscript.

Funding: This work was funded by the Federal Ministry of Education and Research (BMBF) funding measure HyReKA (02WRS1377) and grants of the Bundesinstitut für Risikobewertung (43-001 and 1322-648), European Joint Programme One Health EJP (ARDIG and Full_Force) and the BMG project GÜCCI.

Institutional Review Board Statement: Not applicable.

Informed Consent Statement: Not applicable.

Data Availability Statement: The data for this study were deposited in the Sequence Read Archive (SRA) at NCBI under accession number PRJNA706398.

Acknowledgments: We thank the staff of the participating slaughterhouses for their kind cooperation.

Conflicts of Interest: The authors declare no conflict of interest. The funders had no role in study design, data collection and interpretation, or the decision to submit the work for publication.

\section{References}

1. Federal Ministry of Food and Agriculture. Report of the Federal Ministry of Food and Agriculture on the Evaluation of the Antibiotics Minimisation Concept introduced with the 16th Act to Amend the Medicinal Products Act (16th AMG Amendment): Evaluation based on section 58g of the Medicinal Products Act. Available online: https://www.bmel.de/SharedDocs/ Downloads/EN/_Animals/Report-16thAMGAmendment.pdf?_blob=publicationFile\&v=4 (accessed on 23 February 2021).

2. EMEA. Antibiotic Resistance in the European Union Associated with Therapeutic Use of Veterinary Medicines: Report and Quantitative Risk Assessment by the Committee for Veterinary Medicinal Products. Available online: https://www.ema.europa.eu/en/ documents/report/antibiotic-resistance-european-union-associated-therapeutic-use-veterinary-medicines-report_en-0.pdf (accessed on 23 February 2021).

3. European Centre for Disease Prevention and Control (ECDC). The European Union Summary Report on Antimicrobial Resistance in zoonotic and indicator bacteria from humans, animals and food in 2017/2018. EFSA J. 2020, 18, e06007. [CrossRef]

4. Ghodousi, A.; Bonura, C.; Di Noto, A.M.; Mammina, C. Extended-Spectrum B-Lactamase, AmpC-Producing, and FluoroquinoloneResistant Escherichia coli in Retail Broiler Chicken Meat, Italy. Foodborne Pathog. Dis. 2015, 12, 619-625. [CrossRef] [PubMed]

5. Blaak, H.; Van Hoek, A.H.A.M.; Hamidjaja, R.A.; van der Plaats, R.Q.; Kerkhof-de Heer, L.; de Roda Husman, A.M.; Schets, F.M. Distribution, Numbers, and Diversity of ESBL-Producing E. coli in the Poultry Farm Environment. PLoS ONE 2015, 10, e0135402. [CrossRef] [PubMed]

6. Savin, M.; Bierbaum, G.; Hammerl, J.A.; Heinemann, C.; Parcina, M.; Sib, E.; Voigt, A.; Kreyenschmidt, J. ESKAPE Bacteria and Extended-Spectrum- $\beta$-Lactamase-Producing Escherichia coli Isolated from Wastewater and Process Water from German Poultry Slaughterhouses. Appl. Environ. Microbiol. 2020, 86. [CrossRef]

7. Savin, M.; Bierbaum, G.; Hammerl, J.A.; Heinemann, C.; Parcina, M.; Sib, E.; Voigt, A.; Kreyenschmidt, J. Antibiotic-resistant bacteria and antimicrobial residues in wastewater and process water from German pig slaughterhouses and their receiving municipal wastewater treatment plants. Sci. Total Environ. 2020, 727, 138788. [CrossRef]

8. Schechter, L.M.; Creely, D.P.; Garner, C.D.; Shortridge, D.; Nguyen, H.; Chen, L.; Hanson, B.M.; Sodergren, E.; Weinstock, G.M.; Dunne, W.M.; et al. Extensive Gene Amplification as a Mechanism for Piperacillin-Tazobactam Resistance in Escherichia coli. mBio 2018, 9. [CrossRef] [PubMed]

9. Livermore, D.M.; Day, M.; Cleary, P.; Hopkins, K.L.; Toleman, M.A.; Wareham, D.W.; Wiuff, C.; Doumith, M.; Woodford, N. OXA-1 $\beta$-lactamase and non-susceptibility to penicillin/ $\beta$-lactamase inhibitor combinations among ESBL-producing Escherichia coli. J. Antimicrob. Chemother. 2019, 74, 326-333. [CrossRef] 
10. de Kraker, M.E.A.; Stewardson, A.J.; Harbarth, S. Will 10 Million People Die a Year due to Antimicrobial Resistance by 2050? PLoS Med. 2016, 13, e1002184. [CrossRef]

11. Manges, A.R.; Geum, H.M.; Guo, A.; Edens, T.J.; Fibke, C.D.; Pitout, J.D.D. Global Extraintestinal Pathogenic Escherichia coli (ExPEC) Lineages. Clin. Microbiol. Rev. 2019, 32. [CrossRef] [PubMed]

12. Ewers, C.; Janssen, T.; Wieler, L.H. Aviäre pathogene Escherichia coli (APEC). Berl. Munch. Tierarztl. Wochenschr. 2003, 116, 381-395.

13. Maluta, R.P.; Logue, C.M.; Casas, M.R.T.; Meng, T.; Guastalli, E.A.L.; Rojas, T.C.G.; Montelli, A.C.; Sadatsune, T.; de Carvalho Ramos, M.; Nolan, L.K.; et al. Overlapped Sequence Types (STs) and Serogroups of Avian Pathogenic (APEC) and Human Extra-Intestinal Pathogenic (ExPEC) Escherichia coli Isolated in Brazil. PLoS ONE 2014, 9, e105016. [CrossRef]

14. Mitchell, N.M.; Johnson, J.R.; Johnston, B.; Curtiss, R.; Mellata, M. Zoonotic Potential of Escherichia coli Isolates from Retail Chicken Meat Products and Eggs. Appl. Environ. Microbiol. 2015, 81, 1177-1187. [CrossRef]

15. Dohmen, W.; Van Gompel, L.; Schmitt, H.; Liakopoulos, A.; Heres, L.; Urlings, B.A.; Mevius, D.; Bonten, M.J.M.; Heederik, D.J.J. ESBL carriage in pig slaughterhouse workers is associated with occupational exposure. Epidemiol. Infect. 2017, 145, 2003-2010. [CrossRef] [PubMed]

16. Nde, C.W.; McEvoy, J.M.; Sherwood, J.S.; Logue, C.M. Cross Contamination of Turkey Carcasses by Salmonella Species during Defeathering. Poult. Sci. 2007, 86, 162-167. [CrossRef] [PubMed]

17. Zurfluh, K.; Bagutti, C.; Brodmann, P.; Alt, M.; Schulze, J.; Fanning, S.; Stephan, R.; Nüesch-Inderbinen, M. Wastewater is a reservoir for clinically relevant carbapenemase- and 16s rRNA methylase-producing Enterobacteriaceae. Int. J. Antimicrob. Agents 2017, 50, 436-440. [CrossRef]

18. KRINKO. Hygienemaßnahmen bei Infektionen oder Besiedlung mit multiresistenten gramnegativen Stäbchen. Empfehlung der Kommission für Kranken-haushygiene und Infektionsprävention (KRINKO) beim Robert Koch-Institut (RKI). Bundesgesundh Gesundh. Gesundh. 2012, 55, 1311-1354. [CrossRef] [PubMed]

19. Clermont, O.; Christenson, J.K.; Denamur, E.; Gordon, D.M. The Clermont Escherichia coli phylo-typing method revisited: Improvement of specificity and detection of new phylo-groups. Environ. Microbiol. Rep. 2013, 5, 58-65. [CrossRef]

20. Johnson, J.R.; Gajewski, A.; Lesse, A.J.; Russo, T.A. Extraintestinal Pathogenic Escherichia coli as a Cause of Invasive Nonurinary Infections. J. Clin. Microbiol. 2003, 41, 5798-5802. [CrossRef]

21. Spurbeck, R.R.; Dinh, P.C.; Walk, S.T.; Stapleton, A.E.; Hooton, T.M.; Nolan, L.K.; Kim, K.S.; Johnson, J.R.; Mobley, H.L.T. Escherichia coli Isolates That Carry vat, fyuA, chuA, and yfcV Efficiently Colonize the Urinary Tract. Infect. Immun. 2012, 80, 4115-4122. [CrossRef]

22. Chen, S.; Zhou, Y.; Chen, Y.; Gu, J. fastp: An ultra-fast all-in-one FASTQ preprocessor. Bioinformatics 2018, 34, i884-i890. [CrossRef]

23. Ondov, B.D.; Treangen, T.J.; Melsted, P.; Mallonee, A.B.; Bergman, N.H.; Koren, S.; Phillippy, A.M. Mash: Fast genome and metagenome distance estimation using MinHash. Genome Biol. 2016, 17, 132. [CrossRef]

24. Mikheenko, A.; Prjibelski, A.; Saveliev, V.; Antipov, D.; Gurevich, A. Versatile genome assembly evaluation with QUAST-LG. Bioinformatics 2018, 34, i142-i150. [CrossRef] [PubMed]

25. Feldgarden, M.; Brover, V.; Haft, D.H.; Prasad, A.B.; Slotta, D.J.; Tolstoy, I.; Tyson, G.H.; Zhao, S.; Hsu, C.-H.; McDermott, P.F.; et al. Using the NCBI AMRFinder Tool to Determine Antimicrobial Resistance Genotype-Phenotype Correlations Within a Collection of NARMS Isolates. BioRxiv 2019, 550707. [CrossRef]

26. Chen, L.; Yang, J.; Yu, J.; Yao, Z.; Sun, L.; Shen, Y.; Jin, Q. VFDB: A reference database for bacterial virulence factors. Nucleic Acids Res. 2005, 33, D325-D328. [CrossRef] [PubMed]

27. Jolley, K.A.; Maiden, M.C.J. BIGSdb: Scalable analysis of bacterial genome variation at the population level. BMC Bioinform. 2010, 11, 595. [CrossRef] [PubMed]

28. Bundesamt für Verbraucherschutz und Lebensmittelsicherheit. BVL-Report 13.7. Bericht zur Resistenzmonitoringstudie 2017: Resistenzsituation bei Klinisch Wichtigen Tierpathogenen Bakterien. Available online: https:/ /www.bvl.bund.de/SharedDocs / Berichte/07_Resistenzmonitoringstudie/Bericht_Resistenzmonitoring_2017.html (accessed on 5 March 2021).

29. Bundesamt für Verbraucherschutz und Lebensmittelsicherheit. BVL-Report 15.2. Berichte zur Lebensmittelsicherheit: ZoonosenMonitoring 2019. Available online: https://www.bvl.bund.de/SharedDocs/Downloads/01_Lebensmittel/04_Zoonosen_ Monitoring/Zoonosen_Monitoring_Bericht_2019.pdf?_blob=publicationFile\&v=5 (accessed on 5 March 2021).

30. World Organisation for Animal Health. Oie List of Antimicrobial Agents of Veterinary Importance. Available online: https: //www.oie.int/fileadmin/Home/eng/Our_scientific_expertise/docs/pdf/AMR/A_OIE_List_antimicrobials_May2018.pdf (accessed on 23 February 2021).

31. World Health Organisation. Critically Important Antimicrobials for Human Medicine: 6th Revision 2018. Ranking of Medically Important Antimicrobials for Risk Management of Antimicrobial Resistance due to Non-human Use. Available online: https: / / apps.who.int/iris/bitstream/handle/10665/312266/9789241515528-eng.pdf?ua=1 (accessed on 23 February 2021).

32. European Medicines Agency. Reflection Paper on Use of Aminoglycosides in Animals in the European Union: Development of Resistance and Impact on Human and Animal Health. Available online: https://www.ema.europa.eu/en/documents/scientificguideline/draft-reflection-paper-use-aminoglycosides-animals-european-union-development-resistance-impact_en.pdf (accessed on 23 February 2021).

33. Hidalgo-Grass, C.; Strahilevitz, J. High-Resolution Melt Curve Analysis for Identification of Single Nucleotide Mutations in the Quinolone Resistance Gene aac(6')-Ib-cr. Antimicrob. Agents Chemother. 2010, 54, 3509-3511. [CrossRef] 
34. Weiner, L.M.; Webb, A.K.; Limbago, B.; Dudeck, M.A.; Patel, J.; Kallen, A.J.; Edwards, J.R.; Sievert, D.M. Antimicrobial-Resistant Pathogens Associated With Healthcare-Associated Infections: Summary of Data Reported to the National Healthcare Safety Network at the Centers for Disease Control and Prevention, 2011-2014. Infect. Control Hosp. Epidemiol. 2016, 37, $1288-1301$. [CrossRef] [PubMed]

35. Suetens, C.; Latour, K.; Kärki, T.; Ricchizzi, E.; Kinross, P.; Moro, M.L.; Jans, B.; Hopkins, S.; Hansen, S.; Lyytikäinen, O.; et al. Prevalence of healthcare-associated infections, estimated incidence and composite antimicrobial resistance index in acute care hospitals and long-term care facilities: Results from two European point prevalence surveys, 2016 to 2017. Eurosurveillance 2018, 23, 1800516. [CrossRef]

36. Zhou, K.; Tao, Y.; Han, L.; Ni, Y.; Sun, J. Piperacillin-Tazobactam (TZP) Resistance in Escherichia coli Due to Hyperproduction of TEM-1 $\beta$-Lactamase Mediated by the Promoter Pa/Pb. Front. Microbiol. 2019, 10, 833. [CrossRef]

37. Roer, L.; Overballe-Petersen, S.; Hansen, F.; Schønning, K.; Wang, M.; Røder, B.L.; Hansen, D.S.; Justesen, U.S.; Andersen, L.P.; Fulgsang-Damgaard, D.; et al. Escherichia coliSequence Type 410 Is Causing New International High-Risk Clones. mSphere 2018, 3, e00337-18. [CrossRef]

38. Pietsch, M.; Eller, C.; Wendt, C.; Holfelder, M.; Falgenhauer, L.; Fruth, A.; Grössl, T.; Leistner, R.; Valenza, G.; Werner, G.; et al. Molecular characterisation of extended-spectrum $\beta$-lactamase (ESBL)-producing Escherichia coli isolates from hospital and ambulatory patients in Germany. Vet. Microbiol. 2017, 200, 130-137. [CrossRef]

39. Schaekel, F.; May, T.; Seiler, J.; Hartmann, M.; Kreienbrock, L. Antibiotic drug usage in pigs in Germany-Are the class profiles changing? PLoS ONE 2017, 12, e0182661. [CrossRef] [PubMed]

40. Rosato, A.; Vicarini, H.; Leclercq, R. Inducible or constitutive expression of resistance in clinical isolates of streptococci and enterococci cross-resistant to erythromycin and lincomycin. J. Antimicrob. Chemother. 1999, 43, 559-562. [CrossRef] [PubMed]

41. Zou, L.; Meng, J.; McDermott, P.F.; Wang, F.; Yang, Q.; Cao, G.; Hoffmann, M.; Zhao, S. Presence of disinfectant resistance genes in Escherichia coli isolated from retail meats in the USA. J. Antimicrob. Chemother. 2014, 69, 2644-2649. [CrossRef]

42. Leverstein-van Hall, M.A.; Dierikx, C.M.; Cohen Stuart, J.; Voets, G.M.; van den Munckhof, M.P.; van Essen-Zandbergen, A.; Platteel, T.; Fluit, A.C.; van de Sande-Bruinsma, N.; Scharinga, J.; et al. Dutch patients, retail chicken meat and poultry share the same ESBL genes, plasmids and strains. Clin. Microbiol. Infect. 2011, 17, 873-880. [CrossRef] [PubMed]

43. Borges, C.A.; Tarlton, N.J.; Riley, L.W. Escherichia coli from Commercial Broiler and Backyard Chickens Share Sequence Types, Antimicrobial Resistance Profiles, and Resistance Genes with Human Extraintestinal Pathogenic Escherichia coli. Foodborne Pathog. Dis. 2019, 16, 813-822. [CrossRef]

44. Büdel, T.; Kuenzli, E.; Campos-Madueno, E.I.; Mohammed, A.H.; Hassan, N.K.; Zinsstag, J.; Hatz, C.; Endimiani, A. On the island of Zanzibar people in the community are frequently colonized with the same MDR Enterobacterales found in poultry and retailed chicken meat. J. Antimicrob. Chemother. 2020, 75, 2432-2441. [CrossRef]

45. Blaak, H.; de Kruijf, P.; Hamidjaja, R.A.; van Hoek, A.H.A.M.; de Roda Husman, A.M.; Schets, F.M. Prevalence and characteristics of ESBL-producing E. coli in Dutch recreational waters influenced by wastewater treatment plants. Vet. Microbiol. 2014, 171, 448-459. [CrossRef]

46. Paulshus, E.; Thorell, K.; Guzman-Otazo, J.; Joffre, E.; Colque, P.; Kühn, I.; Möllby, R.; Sørum, H.; Sjöling, Å. Repeated Isolation of Extended-Spectrum- $\beta$-Lactamase-Positive Escherichia coli Sequence Types 648 and 131 from Community Wastewater Indicates that Sewage Systems Are Important Sources of Emerging Clones of Antibiotic-Resistant Bacteria. Antimicrob. Agents Chemother. 2019, 63. [CrossRef]

47. Bleichenbacher, S.; Stevens, M.J.A.; Zurfluh, K.; Perreten, V.; Endimiani, A.; Stephan, R.; Nüesch-Inderbinen, M. Environmental dissemination of carbapenemase-producing Enterobacteriaceae in rivers in Switzerland. Environ. Pollut. 2020, $265,115081$. [CrossRef] [PubMed]

48. Manges, A.R.; Johnson, J.R. Food-Borne Origins of Escherichia coli Causing Extraintestinal Infections. Clin. Infect. Dis. 2012, 55, 712-719. [CrossRef]

49. Aslam, M.; Toufeer, M.; Narvaez Bravo, C.; Lai, V.; Rempel, H.; Manges, A.; Diarra, M.S. Characterization of Extraintestinal Pathogenic Escherichia coli isolated from retail poultry meats from Alberta, Canada. Int. J. Food Microbiol. 2014, 177, 49-56. [CrossRef]

50. Müller, A.; Stephan, R.; Nüesch-Inderbinen, M. Distribution of virulence factors in ESBL-producing Escherichia coli isolated from the environment, livestock, food and humans. Sci. Total Environ. 2016, 541, 667-672. [CrossRef]

51. Khan, S.B.; Zou, G.; Cheng, Y.-T.; Xiao, R.; Li, L.; Wu, B.; Zhou, R. Phylogenetic grouping and distribution of virulence genes in Escherichia coli along the production and supply chain of pork around Hubei, China. J. Microbiol. Immunol. Infect. 2017, 50, 382-385. [CrossRef]

52. Singer, R.S. Urinary tract infections attributed to diverse ExPEC strains in food animals: Evidence and data gaps. Front. Microbiol. 2015, 6, 28. [CrossRef]

53. Poole, N.M.; Green, S.I.; Rajan, A.; Vela, L.E.; Zeng, X.-L.; Estes, M.K.; Maresso, A.W. Role for FimH in Extraintestinal Pathogenic Escherichia coli Invasion and Translocation through the Intestinal Epithelium. Infect. Immun. 2017, 85. [CrossRef]

54. Tseng, C.-C.; Lin, W.-H.; Wu, A.-B.; Wang, M.-C.; Teng, C.-H.; Wu, J.-J. Escherichia coli FimH adhesins act synergistically with PapG II adhesins for enhancing establishment and maintenance of kidney infection. J. Microbiol. Immunol. Infect. 2020. [CrossRef] [PubMed] 
55. Maluta, R.P.; Leite, J.L.; Rojas, T.C.G.; Scaletsky, I.C.A.; Guastalli, E.A.L.; Ramos, M.D.C.; Dias da Silveira, W. Variants of ast A gene among extra-intestinal Escherichia coli of human and avian origin. FEMS Microbiol. Lett. 2017, 364. [CrossRef] [PubMed]

56. Yatsuyanagi, J.; Saito, S.; Miyajima, Y.; Amano, K.-I.; Enomoto, K. Characterization of Atypical Enteropathogenic Escherichia coli Strains Harboring the astA Gene That Were Associated with a Waterborne Outbreak of Diarrhea in Japan. J. Clin. Microbiol. 2003, 41, 2033-2039. [CrossRef]

57. Sarowska, J.; Futoma-Koloch, B.; Jama-Kmiecik, A.; Frej-Madrzak, M.; Ksiazczyk, M.; Bugla-Ploskonska, G.; Choroszy-Krol, I. Virulence factors, prevalence and potential transmission of extraintestinal pathogenic Escherichia coli isolated from different sources: Recent reports. Gut Pathog. 2019, 11, 10. [CrossRef] [PubMed]

58. Manges, A.R.; Dietrich, P.S.; Riley, L.W. Multidrug-ResistantEscherichia coliClonal Groups Causing Community-Acquired Pyelonephritis. Clin. Infect. Dis. 2004, 38, 329-334. [CrossRef] [PubMed]

59. Flores-Mireles, A.L.; Walker, J.N.; Caparon, M.; Hultgren, S.J. Urinary tract infections: Epidemiology, mechanisms of infection and treatment options. Nat. Rev. Microbiol. 2015, 13, 269-284. [CrossRef] [PubMed]

60. Zhi, S.; Stothard, P.; Banting, G.; Scott, C.; Huntley, K.; Ryu, K.; Otto, S.; Ashbolt, N.; Checkley, S.; Dong, T.; et al. Characterization of water treatment-resistant and multidrug-resistant urinary pathogenic Escherichia coli in treated wastewater. Water Res. 2020, 182, 115827. [CrossRef]

61. Zhang, M.; Wan, K.; Zeng, J.; Lin, W.; Ye, C.; Yu, X. Co-selection and stability of bacterial antibiotic resistance by arsenic pollution accidents in source water. Environ. Int. 2020, 135, 105351. [CrossRef] [PubMed]

62. Hasman, H.; Kempf, I.; Chidaine, B.; Cariolet, R.; Ersbøll, A.K.; Houe, H.; Bruun Hansen, H.C.; Aarestrup, F.M. Copper Resistance in Enterococcus faecium, Mediated by the tcrB Gene, Is Selected by Supplementation of Pig Feed with Copper Sulfate. Appl. Environ. Microbiol. 2006, 72, 5784-5789. [CrossRef] [PubMed]

63. Yazdankhah, S.; Rudi, K.; Bernhoft, A. Zinc and copper in animal feed-Development of resistance and co-resistance to antimicrobial agents in bacteria of animal origin. Microb. Ecol. Health Dis. 2014, 25. [CrossRef]

64. Medardus, J.J.; Molla, B.Z.; Nicol, M.; Morrow, W.M.; Rajala-Schultz, P.J.; Kazwala, R.; Gebreyes, W.A. In-Feed Use of Heavy Metal Micronutrients in U.S. Swine Production Systems and Its Role in Persistence of Multidrug-Resistant Salmonellae. Appl. Environ. Microbiol. 2014, 80, 2317-2325. [CrossRef] 